\title{
Wahl des Interventionszeitpunktes bei chronischen Herzklappenfehlern Operationsindikation anhand aktueller Leitlinien
}

Dieter Horstkotte, Cornelia Piper

Kardiologische Klinik, Herz- und Diabeteszentrum NRW, Ruhr Universität Bochum, Bad Oeynhausen

Herzklappenfehler resultieren über die Druck- und/oder Volumenbelastung in morphologischen Veränderungen des Myokards. Die systematische Erfassung von Symptomen mittels Fragebogen vor dem ärztlichen Gespräch hat sich bewährt. Die Objektivierung der Symptomatik erfolgt im Rahmen einer Ergometrie. Für die schwere chronische Mitralinsuffizienz (Regurgitationsfraktion $>45 \%$ ) sehen die aktuellen Leitlinien eine Operationsindikation, sobald die Patienten symptomatisch werden. Bei einer Ejektionsfraktion < $30 \%$ und/oder einem endsystolischen Diameter des linken Ventrikels größer 55 mm werden die Langzeitergebnisse der Mitralklappenchirurgie kritisch gesehen. Auch neu aufgetretenes Vorhofflimmern, ebenso wie ein systolischer Pulmonalarteriendruck > 50 mm in Ruhe oder $>60$ mmHg unter Belastung stellt ein Interventionskriterium dar. Bei jeder manifesten, auf eine Aortenstenose zurückzuführenden myokardialen Insuffizienz (dekompensierte Aortenstenose), bei kausal symptomatischer (Schwindel, Synkopen, Angina pectoris bei Fehlen einer КНК, Arrhythmien), aber myokardial kompensierter Aortenstenose, bei Nachweis progredienter ST-T-Veränderungen im EKG oder stummen Myokardischämien sowie bei asymptomatischen Patienten mit inadäquater myokardialer Adaptation (Abfall der Ejektionsfraktion oder des Schlagvolumens unter Belastung) ist die Indikation zur chirurgischen Intervention unumstritten. Die Indikation zum Aortenklappenersatz besteht gemäß den aktuellen Leitlinien bei schwerer Aortensinsuffizienz, sobald die Patienten symptomatisch werden. Weitere Indikationen zur Operation sind eine LV-Ejektionsfraktion $\leq \mathbf{5 0} \%$ (Evidenzklasse I), ein pathophysiologischer Belastungstest mit Anstieg der linksventrikulären Ejektionsfraktion um weniger als $5 \%$, ein linksventrikulärer endsystolischer Diameter $>\mathbf{5 0} \mathbf{~ m m}$ (Ila) und ein linksventrikulärer enddiastolischer Diameter $>70 \mathrm{~mm}$.

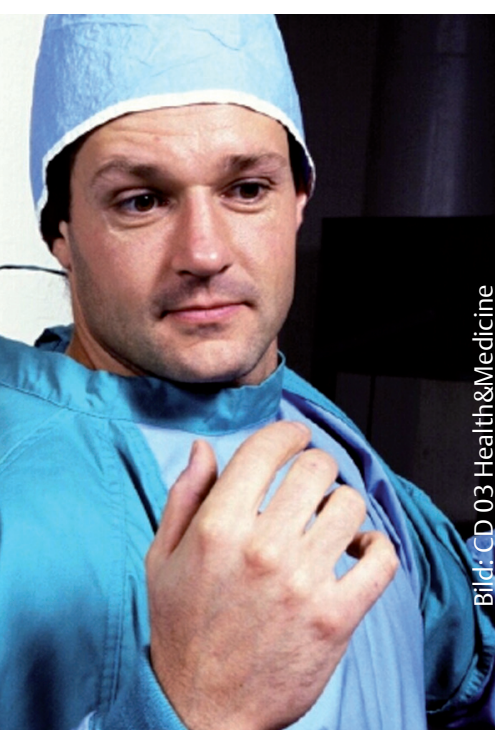
punkt stehen dabei die konzentrische (Aortenstenose) oder exzentrische linksventrikuläre Hypertrophie (Aorten- und Mitralinsuffizienz) bei Mitralklappenfehlern - und die $z$ nächst parallel dem Hypertrophieprozess verlaufende, fibroblastenvermittelte Vermehrung von intrakardialem Bindegewebe (1).

Eine überproportionale intrakardiale Bindegewebsvermehrung resultiert in einer progredienten diastolischen Herzinsuffizienz (linksventrikuläre Compliance-Störung), die durch disseminierte Vernarbungen im Gefolge von Innenschichtischämien insbesondere bei Aortenstenose noch verstärkt wird (2). Nach weitgehender Beseitigung der myokardialen Druck-/Volumenbelastung, z. B. durch einen Klappenersatz, kommt es binnen Wochen bis Monaten zu einer Abnahme der Herzmuskelmasse („Hypertrophieregression“), während die Regression des Bindegewebes im günstigsten Fall einige Jahre in Anspruch nimmt. Auch nach erfolgreicher Klappenintervention nimmt deshalb die diastolische Funktionsstörung oft zu (3). Die dann häufig von Patienten unverändert oder sogar verstärkt angegebene Luftnot ist diesem Remodeling der Myokardstruktur geschuldet, nicht einem unbefriedigenden Interventionsergebnis oder gar einer falschen Indikationsstellung. Durch eine frühe Intervention wären die Probleme größtenteils vermeidbar (4).

\section{Bedeutung und Objektivierung der klinischen Symptomatik}

Über viele Jahrzehnte erfolgten Klappeninterventionen ausschließlich aufgrund der patientenseitig angegebenen Symptomatik. Bis in die jüngeren Leitlinien findet sich diese Differenzierung in "asymptomatische“ und „symptomatische“ Klappenfehler (5, 6). Dies gründet auf wegweisende ältere Untersuchungen zum natürlichen Verlauf z. B. von Patienten mit Aortenklappenstenosen, die eindrucksvoll zeigen konnten, dass nach Manifestation von Symptomen der Herzinsuffizienz, von Synkopen oder Vitien-bedingter Angina-pectoris-Symptomatik die weitere Prognose ohne Operation ausgesprochen limitiert ist $(7,8,9)$. Der Umkehrschluss, beim Fehlen prognoselimitierender Symptome sei der Interventionszeitpunkt, zu dem Lebenserwartung und Lebensqualität am günstigsten beeinflusst werden, noch nicht erreicht, verbietet sich selbstredend.

Die systematische Erfassung von Symptomen 
mittels Fragebogen (Abb. 1) vor dem ärztlichen Gespräch hat sich bewährt. Die Objektivierung der (nicht) berichteten Beschwerdesymptomatik ist unverzichtbar, da sich viele Patienten mit chronischen Herzklappenfehlern an die eingeschränkte Leistungsfähigkeit anpassen und insbesondere betagte Patienten sich bestimmten Belastungen nicht mehr aussetzen („sedentary lifestyle“). Die Objektivierung der Symptomatik erfolgt am besten im Rahmen einer Ergometrie mit Bestimmung der maximalen körperlichen Leistungsfähigkeit, der Sauerstoffaufnahme an der anaeroben Schwelle und der maximalen Sauerstoffaufnahme (Spiroergometrie) (Abb. 2 ). Bei systematischen Untersuchungen zeigt sich dabei, dass die subjektiven Patientenangaben und die objektivierbaren Parameter erheblich differieren (Abb. 3).

\section{Pathophysiologie und Lastadaptation bei Mitralinsuffizienz}

Bei schlussunfähiger Mitralklappe fördert der linke Ventrikel systolisch nach retrograd und nach antegrad Volumen. Die Regurgitation in den linken Vorhof erfolgt überwiegend in der Präejektionsphase, bevor die Aortenklappe öffnet und damit gegen sehr geringen Widerstand. Folglich kann die Kontraktilität des linken Ventrikels (in der Regel gemessen anhand der Ejektionsfraktion) bildgeberisch nur schwer beurteilt werden. Hier sei daran erinnert, dass sich effektive Auswurfleistung und „Kontraktiliät“ nur auf das antegrad, gegen Systemwiderstand, geförderte Blutvolumen bezieht und bei bedeutsamen Regurgitationsvolumina in das Niederdruckgebiet - mit nur dadurch erhaltener Ejektionsfraktion - selbst fortgeschrittene linksventrikuläre Pumpfunktionsstörungen maskiert werden können. Das antregrad fehlende Volumen wird durch Erhöhung des periphervaskulären Widerstandes kompensiert, was wiederum die Regurgitationsfraktion erhöht und den Klappenfehler im Sinne eines Circulus vitiosus voranschreiten lässt $(10,11)$.

\section{Interventionsindikation bei Mitralinsuffizienz}

Die aktuellen Leitlinien $(5,6)$ sehen für die schwere (Regurgitationsfraktion > 45\%) chronische Mitralinsuffizienz eine Operationsindikation, sobald die Patienten symptomatisch werden (Evidenzklasse I). Auf eventuelle Probleme der zuverlässigen Zuordnung von (nicht) berichteten Symptomen und der Notwendigkeit, diese zu objektivieren (Spiroergometrie) wurde bereits hingewiesen. Ebenfalls mit Empfehlung Stärke I ist eine Operation angeraten, bei einem endsystolischen Durchmesser $\geq 40 \mathrm{~mm}$, einer auf 30-60\% reduzierten linksventrikulären Pumpfunktion und bei einem ausbleiben- den Anstieg (< 5\%) der linksventrikulären Ejektionsfraktion unter dynamischer Belastung. Bei einer Ejektionsfraktion < 30\% und/oder einem endsystolischen Diameter des linken Ventrikels größer $55 \mathrm{~mm}$ werden die Langzeitergebnisse der Mitralklappenchirurgie kritisch gesehen

\section{Eigeneinschätzung von Beschwerden}

Klappenfehler:

Bitte kreuzen Sie an, ob und wenn ja wie häufig eine oder mehrere der nachstehenden Symptome bei Ihnen im letzten Jahr aufgetreten sind

\begin{tabular}{|c|c|c|c|}
\hline & nie & selten & häufig \\
\hline Luftnot im Liegen & $\square$ & $\square$ & $\square$ \\
\hline Luftnot bei Belastung & $\square$ & $\square$ & $\square$ \\
\hline Hustenanfälle unter Belastung & $\square$ & $\square$ & $\square$ \\
\hline Schwindelanfälle & $\square$ & $\square$ & $\square$ \\
\hline Ohnmachtsanfälle & $\square$ & Anzahl & $\square \square$ \\
\hline Brustschmerz / Angina pectoris & $\square$ & $\square$ & $\square$ \\
\hline (unangenehmes) Herzklopfen & $\square$ & $\square$ & $\square$ \\
\hline vermehrtes Herzklopfen & $\square$ & $\square$ & $\square$ \\
\hline Ohrensausen & $\square$ & $\square$ & $\square$ \\
\hline Schweißausbrüche & $\square$ & $\square$ & $\square$ \\
\hline (vermehrte) Müdigkeit & $\square$ & $\square$ & $\square$ \\
\hline (vermehrte) Abgeschlagenheit & $\square$ & $\square$ & $\square$ \\
\hline rasche Ermüdbarkeit & $\square$ & $\square$ ja & $\square$ nein \\
\hline Abnahme der Leistungsfähigkeit & $\square$ & $\square$ ja & $\square$ nein \\
\hline Schneller Herzschlag in Ruhe & $\square$ & $\square$ & $\square$ \\
\hline „Klopfen“ in Hals oder Kopf & $\square$ & $\square$ & $\square$ \\
\hline $\begin{array}{l}\text { Blaufärbung von Lippen } \\
\text { oder Fingern }\end{array}$ & $\square$ & $\square$ & $\square$ \\
\hline
\end{tabular}

Wird vom Arzt ausgefüllt:

$\square$ klassisch asymptomatisch $\square$ symptomatisch $\square$ intermediär

Abb. 1 Fragebogen zur Erfassung der von den Patienten subjektiv empfundenen Beschwerden.

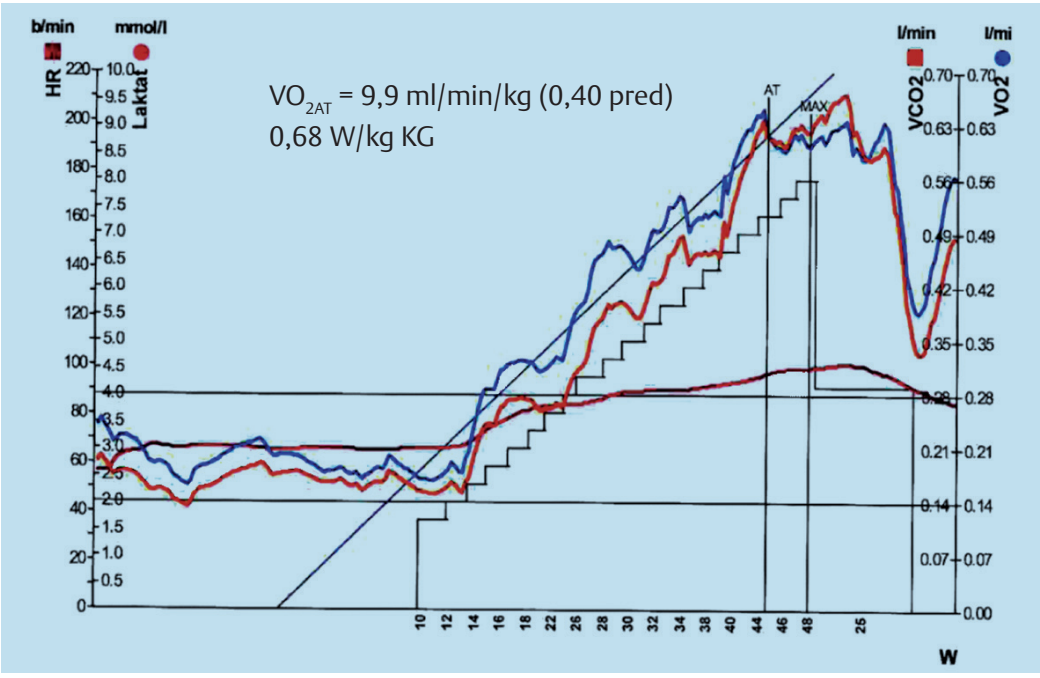

Abb. 2 Spiroergometrie-Befund eines Patienten mit „asymptomatischer“ Aortenstenose. 
und ein hohes Interventionsrisiko unterstellt, sodass eine Operation mit Evidenzklasse Ila nur dann empfohlen wird, wenn der subvalvuläre Klappenapparat erhalten werden kann (11). Letzteres ist bei den derzeit angewandten Operationstechniken allerdings nahezu immer der Fall. Neu aufgetretenes Vorhofflimmern (Evidenzklasse IIa) stellt nach den Leitlinien, ebenso wie ein systolischer Pulmonalarteriendruck $>50 \mathrm{~mm}$ in Ruhe oder $>60 \mathrm{mmHg}$ unter Belastung (IIa) ein Interventionskriterium dar. Es ist unumstritten, dass die Operationsindikation großzügiger gestellt werden kann (z. B. progrediente Dilatation des linken Vorhofs, drohender Verlust des Sinusrhythmus), wenn aufgrund der heute echokardiografisch zuverlässig zu beurteilenden Klappenmorphologie ein klappenerhaltender Eingriff mit hoher Wahrscheinlichkeit möglich ist.

Diese Leitlinien sind jedoch kritisch zu hinterfragen, weil z. B. eine Ejektionsfraktion von nur $50 \%$ ohne jeden Zweifel eine erschöpfte myokardiale Adaptation an die chronische Volumenbelastung anzeigt. Der zuverlässigste Parameter zum Nachweis einer erhaltenen myokardialen Adapation ist die Prüfung, ob bei normaler linksventrikulärer Ejektionsfraktion unter Ruhebedingungen diese unter Belastungsbedingungen um mindestens 5\% ansteigt. Derartige Untersuchungen sind mittels Radionuklidventrikulografie, Magnetresonanztomografie, Stressechokardiografie und auch mit modernen spiroergometrischen Systemen durchführbar. Der ausbleibende Anstieg der linksventrikulären Ejektionsfraktion zeigt nicht nur eine Erschöpfung der myokardialen Adaptationsprozesse an, sondern hat auch nachhaltige prognostische Bedeutung (Abb. 4).

\section{Interventionsindikation \\ bei Mitralstenose}

Therapie der Wahl bei geeigneter Klappenmorphologie ist heute die katheterbasierte Valvoto-

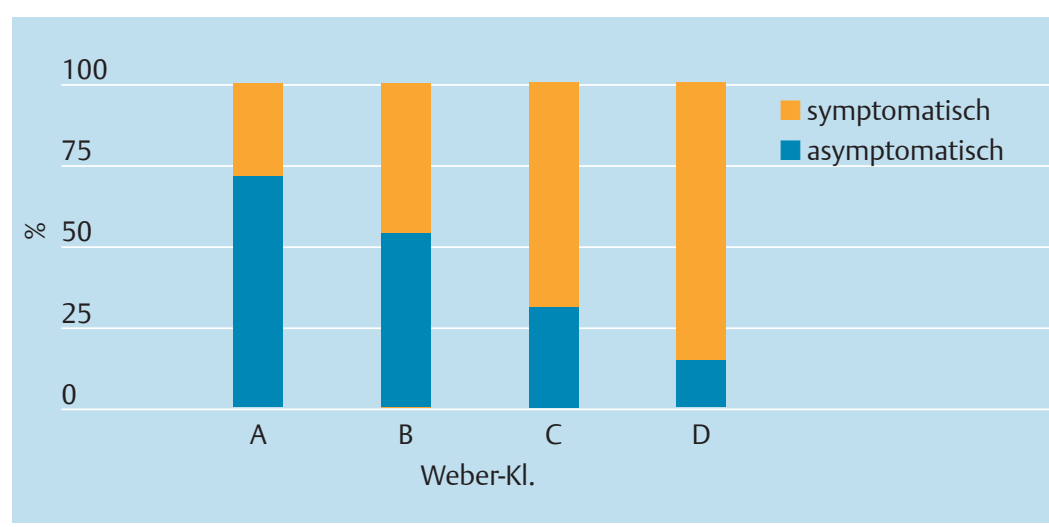

Abb. 3 Subjektive Symptomatik versus Spiroergometrie-Befunde bei fortgeschrittener Aortenstenose (nach standardisiertem Frageprotokoll mit 17 Fragen zu Beschwerden/Symptomen). mie mittels Inoue-Ballon (12). Ist eine Ballonvalvotomie technisch nicht möglich, ist ein Mitralklappenersatz mit Erhalt des subvalvulären Klappenapparates anzustreben.

Für die Langzeitprognose von Patienten mit Mitralstenose sind Dauer und Ausmaß der rechtsventrikulären Druckbelastung entscheidend. Bei rezidivierenden Rechtsherzdekompensationen über einen präoperativen Zeitraum von mehr als 12 Monaten verschlechtert sich die postoperative Prognose signifikant. Dilatationen des rechten Ventrikels über $15 \mathrm{~mm} /$ $\mathrm{m}^{2}$ Körperoberfläche sind ebenfalls bezüglich der Lebenserwartung ungünstig. Die Höhe des durch Druckbelastung und Umbauvorgänge in der Lungenstrombahn erhöhten pulmonal-arteriellen Widerstandes ist dagegen prognostisch nicht prädiktiv, da das Ausmaß einer postoperativen Rückbildung im Einzelfall nicht vorhergesehen werden kann. Wegen der raschen Stenoseprogredienz ab einer Klappenöffnungsfläche $<0,8 \mathrm{~cm}^{2} / \mathrm{m}^{2}$ ist, unabhängig von sonstigen Parametern in dieser Situation, eine Interventionsindikation aus hämodynamischer Sicht gegeben. Gleiches gilt für rezidivierende thromboembolische Ereignisse trotz ausreichender Behandlung mit Vitamin-K-Antagonisten sowie bei Frauen mit höhergradiger Mitralstenose (Mitralklappenöffnungsfläche < 1,5 $\mathrm{cm}^{2}$ ) und beabsichtigter Schwangerschaft.

\section{Pathophysiologie und Lastadaptation bei Aortenstenose}

Die Aortenstenose ist hämodynamisch durch eine linksventrikuläre Nachlasterhöhung mit adaptiver Hypertrophie charakterisiert. Die konsekutive Zunahme der linksventrikulären Muskelmasse zu Lasten des Kavums resultiert in einer konzentrischen Hypertrophie (normale Herzgröße im Thorax-Röntgenbild), sodass bei unveränderter Form des Ventrikelkavums der enddiastolische Ventrikelradius abnimmt. Aufgrund der Hypertrophie stellt sich zunächst eine diastolische Ventrikelfunktionsstörung ein, während die linksventrikuläre systolische Wandspannung weitgehend normal bleibt. Die dadurch bedingte Abnahme des linksventrikulären Schlagvolumens, der Koronarreserve und der frühdiastolischen Koronarperfusion manifestieren sich zunächst in einer myokardialen Ischämie der subendokardialen Wandabschnitte (Kammerendteilveränderungen in den linkspräkordialen EKG-Ableitungen) (2). Durch Utilisation der Vorlastreserve und sympathikusvermittelter Steigung der Kontraktilität ist die systolische linksventrikuläre Pumpfunktion in diesem Stadium der Erkrankung nicht messbar gestört. Die hormonelle Aktivierung, insbesondere des Renin-Angiotensin-Aldosteron-Systems, hat ein Fibroblasten-vermitteltes Remo- 
deling der Kollagenmatrix mit einer besonderen Zunahme von dünnen, elastischen Kollagen-Typ-III-Fasern aber auch von dicken, steifen Kollagen-Typ-I-Fasern, sowie einer ausgeprägten Quervernetzung der Kollagenfasern zur Folge (1).

Ausgeschöpfte Vorlastreserve, Manifestation eines Afterload-Mismatch, Abnahme der Koronarreserve und Veränderung insbesondere des frühdiastolischen Koronarflusses zeigen an, dass die myokardiale Adaptation an die chronische Druckbelastung erschöpft ist. Besteht die Aortenstenose weiter, resultiert regelhaft eine linksventrikuläre Dilatation, bei der sich die Myokardmasse auf einen größeren Radius verteilt (inadäquate, exzentrische Hypertrophie), sodass die systolische linksventrikuläre Wandspannung abrupt ansteigt und die über Jahre oder gar Jahrzehnte weitgehend asymptomatischen Patienten plötzlich symptomatisch werden oder sogar myokardial dekompensieren.

\section{Interventionsindikation}

\section{bei Aortenstenose}

Bei jeder manifesten, auf eine Aortenstenose (AS) zurückzuführenden myokardialen Insuffizienz (dekompensierte Aortenstenose), bei kausal symptomatischer (Schwindel, Synkopen, Angina pectoris bei Fehlen einer KHK, Arrhythmien), aber myokardial kompensierter Aortenstenose, bei Nachweis progredienter STT-Veränderungen im EKG oder stummen Myokardischämien sowie bei asymptomatischen Patienten mit inadäquater myokardialer Adaptation (Abfall der Ejektionsfraktion oder des Schlagvolumens unter Belastung) ist die Indikation zur Intervention unumstritten. Wirklich asymptomatische Patienten mit hochgradiger Aortenstenose tragen ein niedriges Risiko des plötzlichen Herztodes, solange sie sich körperlich schonen. Allerdings beträgt das Risiko, innerhalb von 2 Jahren bedeutsame kardiale Komplikationen zu erleiden oder zu versterben, mehr als $25 \%$, sodass in der Mehrzahl der Fälle die Progression der Erkrankung rasch und das symptomfreie Intervall kurz ist (2). Die Progression der valvulären Aortenstenose ist gut abschätzbar (13).

Jede hämodynamisch bedeutsame Aortenstenose (Klappenöffnungsfläche $<1 \mathrm{~cm}^{2}$ ) kann ohne Vorwarnung symptomatisch werden. Für eine verantwortungsvolle Verlaufsbeobachtung ist es deshalb erforderlich, frühzeitig eine beginnende Erschöpfung der Adaptation an die chronische Druckbelastung zu erkennen, um die Patienten zum optimalen Zeitpunkt einer Intervention zuzuführen. Die Einschränkung der Belastungstoleranz aufgrund einer diastolischen myokardialen Funktionsstörung ist dabei oft wegweisend. Mit Belastungsuntersuchun-

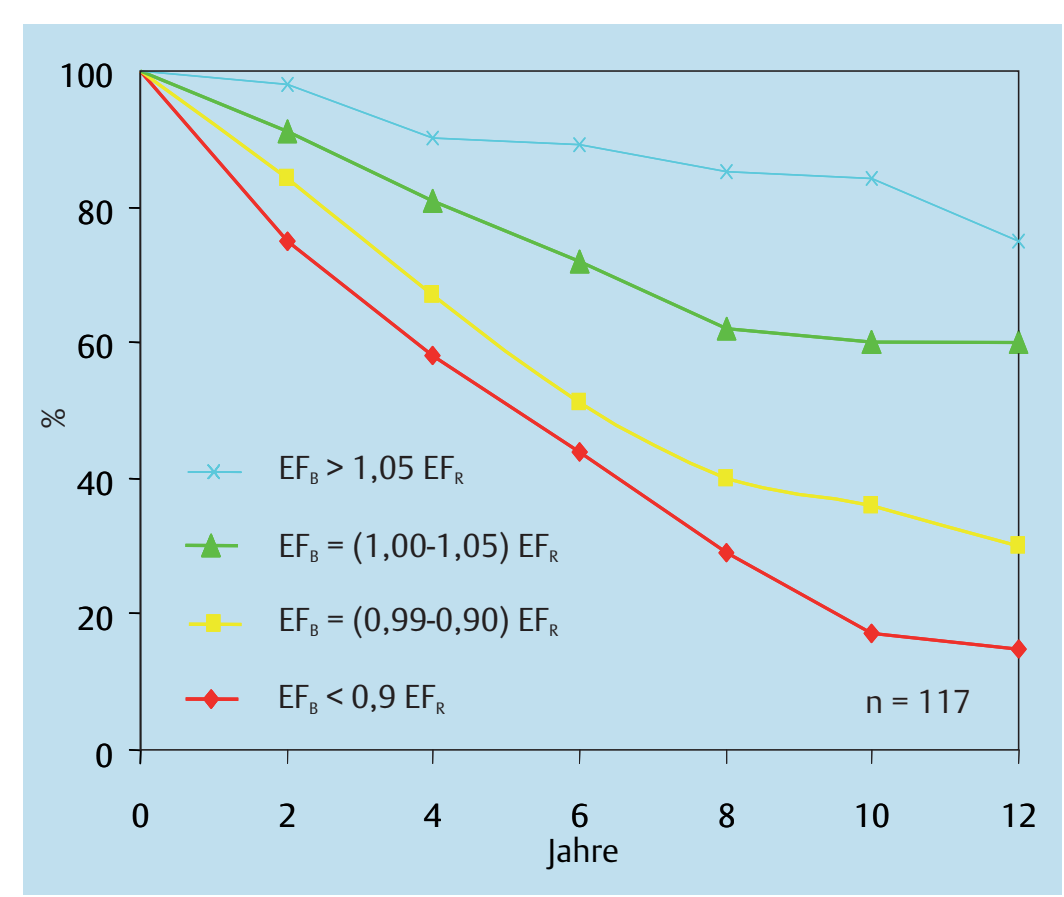

Abb. 4 Einfluss der präoperativ mittels Radionuklidventrikulografie gemessenen Änderung der Ejektionsfraktion unter ergometrischer Belastung (EFB) im Vergleich zu der in Ruhe ermittelten Ejektionsfraktion (EFR) auf die kumulativen Überlebensraten von 117 Patienten mit chronischer Mitralinsuffizienz während einer Nachbeobachtungszeit von 12 Jahren.

gen, die komplikationsfrei durchgeführt werden können, solange alle Kriterien einer unstrittigen Interventionsindikation fehlen, kann eine beginnende Erschöpfung der myokardialen Adaptation frühzeitig erfasst werden. Die Techniken hierfür entsprechen denen, wie sie für die Mitralinsuffizienz besprochen wurden. Die Prognose sowohl der akut als auch der chronisch myokardial dekompensierten Aortenstenose ist außerordentlich schlecht. Diese Patienten haben auch ein hohes Risiko bei konventionellem Aortenklappenersatz, sodass sie primär Kandidaten für einen transfemoralen oder transapikalen perkutanen Klappenersatz sind (14).

\section{Pathophysiologie und Interventionsindi- kation bei Aorteninsuffizienz}

Die hämodynamischen Konsequenzen einer Aorteninsuffizienz sind abhängig von der Regurgitationsfläche, dem peripheren Widerstand, der Diastolendauer und der linksventrikulären Dehnbarkeit. Das ansteigende enddiastolische Ventrikelvolumen resultiert in einer zunehmenden Dilatation und exzentrischen Hypertrophie des linken Ventrikels, mit Verschiebung der Druck-Volumen-Kurve nach rechts, sodass trotz hochgradiger Regurgitation der linksventrikuläre Füllungsdruck lange konstant bleiben kann (adäquate Adaptation) und die Patienten über Jahre asymptomatisch bleiben können. Hierzu trägt auch bei, dass unter 
Belastung aufgrund der frequenzabhängigen Verkürzung der Diastolendauer und der abfallenden systemischen Widerstände die Regurgitationsfraktion am totalen Schlagvolumen abnimmt.

Die Indikation zum Aortenklappenersatz besteht gemäß den aktuellen Leitlinien $(5,6)$ bei schwerer Aortensinsuffizienz, sobald die Patienten symptomatisch werden (Evidenzklasse I). Weitere Indikationen zur Operation sind eine LV-Ejektionsfraktion $\leq 50 \%$ (Evidenzklasse I), ein pathologischer Belastungstest mit Anstieg der linksventrikulären Ejektionsfraktion um weniger als 5\% (I), ein linksventrikulärer endsystolischer Diameter > $50 \mathrm{~mm}$ (IIa) und ein linksventrikulärer enddiastolischer Diameter > $70 \mathrm{~mm}$ (Ila). Die Weite der Aorta aszendens ist bei der Indikationsstellung zum Aortenklappenersatz oder zum klappenerhaltenden Ersatz / Rekonstruktion der Aorta aszendens mit zu berücksichtigen. Allgemein besteht eine Operationsindikation bei einer Weite der Aorta ascendens von $\geq 55$ mm (IIa), bei Patienten mit bikuspider Aortenklappe bei einer Weite der Aorta aszendens $\geq 50 \mathrm{~mm}$ (IIa) und bei

When to intervene in cases of chronic valvular defects - Indications for surgery according to update guidelines

Morphological myocardial changes affect either the pressure exercised on the myocardium or its volume. This causes valvular defects. Before the physician goes into details by questioning the patient, it has proved useful to complete a questionnaire which systematically explores all the symptoms. The symptoms are object ergometrically. As far as severe chronic mitral insufficiency (mitral regurgitation, the regurgitation fraction exceeding $45 \%$ ) is concerned, current guidelines indicate surgery as soon as the patients become symptomatic. The long-term results of mitral valve surgery are seen with a critical eye if the ejection fraction is less than $30 \%$ and/or if the end systolic diameter of the left ventricle is larger than $55 \mathrm{~mm}$. Criteria for intervention are also a newly observed atrial fibrillation as well as a systolic pulmonary artery pressure higher than $50 \mathrm{~mm}$ at rest or greater than $60 \mathrm{~mm}$ under exercise. Indication for surgery is beyond doubt in the following cases: in any manifest myocardial insufficiency due to stenosis of the aorta (decompensated aortic stenosis); in case of a symptomatic but myocardially compensated aortic stenosis (dizziness, syncopes, angina pectoris if there is no cororonary heart disease, arrhythmias); if there are progression ST-T changes in the ECG, or mute myocardial ischemias, as well as in asymptomatic patients with inadequate myocardial adaptation (reduced ejection fraction or reduced stroke volume during exercise). The update guidelines state that aortic valve replacement is indicated in case of severe aortic insufficiency as soon as the patients become symptomatic. Further indications for surgery are an LV ejection fraction equal to or greater than $50 \%$ (evidence class I); a pathophysiological exercise test with an increase of the left ventricular ejection fraction less thant $5 \%$, a left ventricular end systolic diameter greater than $50 \mathrm{~mm}$ (IIa) and a left ventricular end diastolic diameter greater than $70 \mathrm{~mm}$.

\section{Key words}

Valvular defects - intervention - indication for surgery - mitral insufficiency - mitral stenosis - aortic stenosis (aortactia) - aortic insufficiency
Patienten mit Marfan-Syndrom bereits bei Dimensionen von $\geq 45 \mathrm{~mm}$ (I).

\section{Literatur}

1 Piper C, Schultheiss HP, Akdemir D, Rudolf J, Horstkotte D, Pauschinger M. Remodeling of the cardiac extracellular matrix differs between volume- and pressure-overloaded ventricles and is specific for each heart valve lesion. J Heart Valve Dis 2003; 12: 592-600

2 Hering D, Piper C, Horstkotte D. Influence of atypical symptoms and electrocardiographic signs of left ventricular hypertrophy or ST-segment/T-wave abnormalities on the natural history of otherwise asymptomatic adults with moderate to severe aortic stenosis: preliminary communication. J Heart Valve Dis 2004; 13: 182-187

3 Villari B, Vassalli G, Monrad ES et al. Normalization of diastolic dysfunction in aortic stenosis late after valve replacement. Circulation 1995; 91: 2353-2358

4 Horstkotte D, Piper C. Management von Herzklappenpatienten 2008: Was hat sich in den letzten drei Jahrzehnten geändert? Dtsch Med Wochenschr 2008; 133: 280-294

5 Bonow RO, Carabello BA, Chatterjee $\mathrm{K}$ et al. ACC/ AHA 2006 Guidelines for the Management of Patients with Valvular Heart Disease. A Report of the American College of Cardiology/American Heart Association Task Force on Practice Guidelines. J Am Coll Cardiol 2006; 48: 1-148

6 Vahanian A, Baumgartner H, Bax J et al. Guidelines on the management of valvular heart disease. The Task Force on the Management of Valvular Heart Disease of the European Society of Cardiology. Eur Heart J 2007; 28: 230-268

7 Ross J, Braunwald E. Aortic stenosis. Circulation 1968; 38 (Suppl 1): 61-67

8 Horstkotte D, Niehues R, Strauer BE. Pathomorphological aspects, aetiology and natural history of acquired mitral valve stenosis. Eur Heart J 1991; 12 (Suppl. B): 55-60

9 Horstkotte D, Loogen F. The natural history of aortic valve stenosis. Eur Heart ] 1988; 9 (Suppl E): 57-64

10 Piper C, Wiemer M, Schultheiß HP, Horstkotte D. Sinnvolle Diagnostik und Therapieplanung bei organischer und relativer Mitralklappeninsuffizienz. Herz 1998; 23: 429-433

11 Schulte HD, Horstkotte D, Klein M et al. Erhalt des posterioren Mitralsegels und Einfluß auf das Spätergebnis nach Mitralklappenprothesen-Implantation. Z Herz-, Thorax-, Gefäßchir 1992; 6: 11-16

12 Neumayer U, Schmidt HK, Fassbender D, Mannebach $\mathrm{H}$, Bogunovic N, Horstkotte D. Early (three-month) results of percutaneous mitral valvotomy with the Inoue balloon in 1,123 consecutive patients comparing various age groups. Am J Cardiol 2002; 90: 190193

13 Piper C, Bergemann R, Schulte HD, Körfer R, Horstkotte $D$. Ist die Abschätzung der Progression valvulärer Aortenstenosen möglich? Dtsch Med Wochenschr 2000; 125: 484-488

14 Figulla HR, Cremer ], Walther T et al. Positionspapier zur kathetergeführten Aortenklappenintervention. Kardiologe 2009; 3: 199-206

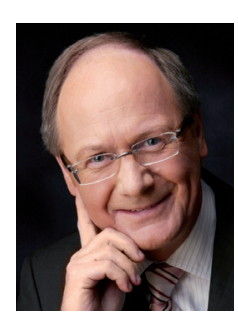

Korrespondenz Univ.-Prof. Dr. med. Dieter Horstkotte Kardiologische Klinik Herz- und Diabeteszentrum NRW Ruhr-Universität Bochum Georgstraße 11 32545 Bad Oeynhausen Fax: 05731/97-2194 akohlstaedt@hdz-nrw.de 